\title{
Comparison of efficacy and safety of oxycodone versus fentanyl for intravenous patient-controlled analgesia in postoperative pain management: a systematic review and meta-analysis
}

\author{
Peng Chen \\ Wuhan University Renmin Hospital \\ Fuchao Chen \\ Dongfeng General Hospital \\ Jiexin Lei \\ Wuhan University renmin Hospital \\ zhou benhong ( $\boldsymbol{\sim}$ benhongzh@whu.edu.cn) \\ Wuhan University Renmin Hospital https://orcid.org/0000-0002-6129-0182
}

Research article

Keywords: oxycodone; fentanyl; patient-controlled analgesia; meta-analysis

Posted Date: July 26th, 2019

DOl: https://doi.org/10.21203/rs.2.11978/v1

License: (a) (1) This work is licensed under a Creative Commons Attribution 4.0 International License.

Read Full License 


\section{Abstract}

Backgroud Patient-controlled analgesia(PCA) with intravenous an opioid has been suggested as a effective method of postoperative pain relief. There are several randomized controlled trials (RCTs) of comparison of oxycodone and fentanyl for IV-PCA in surgical patients. The purpose of this study was to performed a meta-analysis to compare the effect of intravenous oxycodone and fentanyl on postoperative pain in sugical patients from collected current data. Methods The RCTs of oxycodone versus fentanyl for IV-PCA were gathered from PubMed, Embase, Cochrane library, CNKI and VIP data. After data extraction and quality assessment of the included RCTs, the RevMan 5.3 software was applied for metaanalysis of numerical rating scale (NRS) scores, accumulated IV-PCA consumption of oxycodone and fentanyl, patient satisfaction, postoperative nausea and vomiting (PONV), and other adverse events (AEs). Results Results reported from eight RCTs involving 600 patients are included in the meta-analysis. The NRS score at rest and upon movement of group oxycodone was significantly lower than that of group fentanyl $(\mathrm{WMD}=-3.85,95 \% \mathrm{Cl}-4.93 \sim-2.76, \mathrm{P}<0.00001$; WMD $=-4.31,95 \% \mathrm{Cl}-5.79 \sim-2.84$, $\mathrm{P}<0.00001)$; however, the incidence of PONV and dizziness was significantly higher in group oxycodone than in group fentanyl $(\mathrm{OR}=2.41,95 \% \mathrm{Cl} 1.60 \sim 3.63, \mathrm{P}<0.0001 ; \mathrm{OR}=3.69,95 \% \mathrm{Cl} 2.17 \sim 6.26, \mathrm{P}<0.00001)$. Accumulated IV-PCA consumption in group oxycodone was less than in group fentanyl overall the 48 hours postoperatively (WMD $=-12.11,95 \% \mathrm{Cl}-18.42 \sim-5.80, \mathrm{P}=0.0002)$. There was no significant difference in patient satisfaction between oxycodone and fentanyl (OR=0.73, 95\% $\mathrm{Cl} 0.11 \sim 5.04, \mathrm{P}=0.75)$. Conclusion According to the evidence, this meta-analysis suggest that oxycodone IV-PCA is superior to fentanyl in postoperative pain relief, whereas the higher incidence of PONV and dizziness was accompanied with oxycodone. Further large-scale and well-designed studies are needed to summarize and analyse the data to draw a more convincing conclusion.

\section{Background}

Opioids are known to be the most commonly-used medications to manage postoperative pain. ${ }^{1,2}$ However, it is difficult for surgical patients to take drugs orally to relieve pain. On the contrary, administration of intravenous analgesics are very appropriate. ${ }^{3,4}$ patient-controlled intravenous analgesia (PCIA), which are mixed with many drugs in a small container and driven by a syringe for delivery, has been commonly used for the management of postoperative pain in clinical for many years. ${ }^{5,6}$ Patientcotrolled intravenous analgesia ( $\mathrm{PCIA}$ ) with intravenous opioid is a convenient treatment program for postoperative pain, and widely used in clinical practice, because it provides great advantages in improving the prognosis quality of patients and alleviating the economic cost. ${ }^{7,8}$

Dihydrohydroxycodeinone (oxycodone) is a semisynthetic thebaine derivative $\mu$-opioid receptor agonist, and is commonly used to control of moderate to severe pain. ${ }^{9}$ There have been several studies on the efficacy and side effects of oxycodone and it has been proved to have the same potency with morphine. ${ }^{10}$ In recent years, the use of intravenous(IV) oxycodone has increased markedly, and the IV oxycodone for IV-PCA was recently approved by Ministry of Food and Drug Safety(MFDS) in 2013. ${ }^{11}$ 
Consequently, clinical experience regarding the efficacy of oxycodone in IV-PCA is limited. Fentanyl is an opioid analgesic most commonly used for management of postoperative IV-PCA in Korea. ${ }^{12}$ Various studies using fentanyl IV-PCA have revealed that it has extremely high lipid solubility, which results in shortening analgesic onset time on the effect of PCA. ${ }^{13,14}$ Due to the clinical benefits of oxycodone and fentanyl, the use of patient-controlled oxycodone and fentanyl after major surgery is gaining popularity.

Recently, there are several published studies concentrated on this strategy, and have performed a comparison of the analgesic efficacy and tolerability between oxycodone and fentanyl for IV-PCA in postoperative analgesia, but the sample sizes are relatively not sufficient and the results were inconclusive. ${ }^{15}$ Thus, the evidence supporting oxycodone can be an alternative to fentanyl still remains to be controversial and lacks of systemic evaluation of methodologic quality. This meta-analysis was conducted to make a systemic review and meta-analysis about RCTs focused on comparing the efficacy and safety of oxycodone and fentanyl for IV-PCA in postoperative pain management.

\section{Methods}

\section{Data sources and literature search}

We did a systematic review and meta-analysis to perform a comparison of efficacy and safety of oxycodone versus fentanyl for IV-PCA in postoperative pain management. Two reviewers (Chen P and Chen FC) independently searched the medical literature for relevant clinical trials using the electronic databases of PubMed, Cochrane Library, EMBASE, CBMdisc, CNKI, WKSP, and CSJFT, until August, 2018. This was supplemented by searching the reference lists of all retrieved studies, review articles, abstracts and conference reports. The key words used in this search were as follows: "oxycodone", "fentanyl", "patient-controlled analgesia", and "surgical patients" as well as "clinical trials". There were no language restrictions.

\section{Study selection}

Clinical trials that met the following criteria were included: (1) randomized, controlled trials or cohort studies; (2) prospective clinical trials of oxycodone versus fentanyl for IV-PCA; (3) data available for the postoperative pain-related outcomes and adverse events from patients. The exclusion criteria were as follows: (1) case series or single-arm trials; (2) nonrandomized trials; (3) oxycodone and fentanylused used for IV-PCA rather than for anesthesia intraoperatively after surgery; (4) original studies that meet criterion (3) but lacked information such as such as numerical rating scale (NRS) scores, accumulated IVPCA consumption of oxycodone and fentanyl, patient satisfaction and adverse reactions were not available.

\section{Data extraction and quality evaluation}

We extracted from the included trials the following information: the first author, year of publication, number of patients enrolled in the study, treatment proposal and types of surgical operation information 
to understand the baseline of all included studies. Outcomes variable including: NRS scores at 6 timepoints (postoperative 4, 6, 8, 12, 24 and $48 \mathrm{~h}$ ); cumulative PCA dose; patient satisfaction; the proportion of opioid-related adverse events (postoperative nausea and vomiting (PONV), dizziness, headache, pruritis, sedation).

We evaluated the methodological quality of the included studies, according to the following RCT quality evaluation standards of the Cochrane review manual 5.3.0: (1) generation of the random allocation scheme; (2) allocation concealmment; (3) blinding of participants and personnel; (4) blinding of outcome assessment; (5) incomplete outcome data; (6) selective reporting. The risk of bias of each study can be summarized as "high", "low" and "unclear". Any disagreement was settled by discussion or negotiation.

\section{Definition of outcomes}

NRS is a pain rating scale to evaluated the severity of the pain. ${ }^{17}$ An NRS allows a person to describe the intensity of his/her pain as a number usually ranging from 0 to 100 , where "0" corresponds to "no pain" and "100" to "worst pain imaginable." ${ }^{19}$ And NRS scores can be divided into two categories (at rest and movement). The pain observed in activities belongs to pain with movement. ${ }^{20}$ Patients were asked to rank their satisfaction, graded as very satisfied, satisfied, neutral, and dissatisfied. ${ }^{21}$

\section{Statistical analysis}

The statistical analysis was performed with Review Manager Version 5.3 software, which was provided by the Cochrane Collaboration, Oxford, UK. The effect size of categorical outcomes was determined by the pooled odds ratio (OR), along with $95 \%$ confidence intervals $(\mathrm{Cls})$. The continuous results were calculated by weighted mean differences (WMD) with $95 \% \mathrm{Cl}$. The between-study heterogeneity was assessed using the Chi-squared test. If $R$ was $<50 \%(P>0.1)$, the fixed effect model was used; if not $(R>$ $50 \%, P<0.1)$, the random effect model was employed, and we attempted to discover the cause of the heterogeneity. Egger's test was used to evaluate the presence of publication bias. $P$ values less than 0.05 or 0.01 were considered significant. All analyses were based on previous published studies, and no ethics approval or patient consent was required.

\section{Results}

\section{Search results}

A total of 159 documents were obtained through the preliminary examination of the database. And 21 potential literatures were considered eligible by reading the title and abstract. After analyzing the full-text articles, 13 studies which did not meet the inclusion criteria were excluded, and $8^{16-23}$ were found eligible for inclusion according to our criteria for being ultimately included in the Meta-analysis. The selection procedure was described in Fig. 1 which illustrated how the 8 studies were obtained. The 8 selected studies, comprising a total of 600 surgical patients (Oxycodone group: 297 patients; Fentanyl group: 303 patients), all were RCTs investigating the use of oxycodone compared with fentanyl alone for a 
postoperative IV-PCA strategy. Across the all studies, the mean age of patients ranged from 45.6 to 57.8 years for the IV- PCA, with the majority ( $>60 \%$ ) being female from the three available studies. Of the 8 studies, one study ${ }^{19}$ compared the oxycodone and fentanyl for IV-PCA up to postoperative 24 hours, and other seven trails ${ }^{16-18,20-23}$ were 48 hours. The dose of analgesics and surgery type varied among trials, and the main characteristics of included trials are listed in Table 1. The risk assessment of the included studies are shown in Fig. 2 and Fig. 3. Four trials $17,18,20,22$ detailed the methods of randomization, 5 trials ${ }^{18-22}$ detailed the methods of double-blinding, and 2 trials ${ }^{19,22}$ clearly reported allocation concealment.

\section{Meta-analysis of NRS scores}

The WMDs in patient-reported NRS scores at rest were comparable with significant differences at postoperative 8 and 12h between oxycodone and fentanyl for IV-PCA(Fig. 4). For NRS scores at movement, the WMD decreased from $-12.76(95 \% \mathrm{Cl}:-25.15$ to $-0.37, P=0.04)$ at postoperative $4 \mathrm{~h}$ to $-1.04(95 \% \mathrm{Cl}:-1.42$ to -0.66$), P<0.00001)$ at postoperative $12 \mathrm{~h}$, and increased to $-3.30(95 \% \mathrm{Cl}:-5.66$ to $-0.94), P=0.006$ ) at postoperative $24 \mathrm{~h}$ (Fig. 5). The results for the NRS scores of IV-PCA at all time-points in the included trials have been shown in Fig. 4 and Fig. 5.

\section{Meta-analysis of cumulative PCA dose over 48hours}

Data reporting median consumption of infused PCA over 48 hour are described in 6 trials ${ }^{16-19,21,22}$ with 475 patients. Overall, the meta-analysis showed that the amount of PCA overall the 48 hours postoperative after surgery was significantly less in group oxycodone than in group fentanyl (WMD= $-12.11,95 \% \mathrm{Cl}-18.42 \sim-5.80, \mathrm{Z}=3.76, P=0.0002$, Fig. 6 ), using the random-effects model (heterogeneity test, $\left.\mathrm{Chi}^{2}=113.38, \mathrm{df}=5[P<0.0001], \mathrm{I}^{2}=96 \%\right)$.

\section{Meta-analysis of incidence of PONV}

Data on comparisons of the incidence of PONV of oxycodone versus fentanyl were reported by 6 trials ${ }^{17,18,20-23}$ with 855 patients. The results of our fixed-effects $\left(\mathrm{Chi}^{2}=10.31, \mathrm{df}=10[P=0.41], \mathrm{I}^{2}=3 \%\right)$ meta-analysis in terms of PONV were summarized in Fig. 7. There was a higher incidence of PONV $(O R=$ $2.41,95 \% \mathrm{Cl}: 1.60 \sim 3.63, P<0.0001)$ in patients receiving IV-PCA with oxycodone compared with patients receiving fentanyl. Subgroup analysis showed that oxycodone versus fentanyl IV-PCA resulted a significant difference in nauase $(\mathrm{OR}=2.78,95 \% \mathrm{Cl}: 1.75 \sim 4.43, P<0.0001)$, while no significant difference in vomiting was observed between in the two groups (OR=1.43, 95\%Cl: $0.60 \sim 3.44, P=0.42$ ).

\section{Meta-analysis of patient satisfaction for IV-PCA}

The analysis of overall satisfaction in patients for pain management at 48 hours after the surgery was carried in 4 studies ${ }^{17,20-22}$ with 324 patients. Obvious heterogeneity $\left(\mathrm{Chi}^{2}=9.36, \mathrm{df}=3[P=0.03], \mathrm{I}^{2}=68 \%\right)$ was found in the all current included studies. The estimated OR of patient satisfaction for oxycodone 
versus fentanyl IV-PCA during the 48 hours after surgery was 0.73 (95\% Cl: $0.11 \sim 5.04)$ using fixed-effects model, but there was no significant difference ( $P=0.75$, Fig. 8).

\section{Meta-analysis of main adverse events}

For the safety analysis, we selected several of the most frequent AEs. All the meta-analysis results regarding $A E s$ showed that the no obvious difference was observed between oxycodone and fentanyl group in main adverse events (headache: $\mathrm{OR}=1.40,95 \% \mathrm{Cl}[0.69,2.83], P=0.35$; pruritis: $\mathrm{OR}=1.60$, $95 \% \mathrm{Cl}[0.67,3.81], P=0.29$; sedation: $\mathrm{OR}=1.52,95 \% \mathrm{Cl}[0.76,3.04], P=0.24$, but the significant increase of dizziness with oxycodone versus fentanyl was found ( $\mathrm{OR}=3.69,95 \% \mathrm{Cl}[2.17,6.26], P<0.00001)$ (Fig. 9). There was no report of other adverse events including rash, diarrhea, or bradycardia in either group.

\section{Risk of Bias in Included Studies}

Review Manager 5.3 software was used to assess the publication bias by funnel plot, and the details of funnel plot was presented in Fig. 10. As a result, we can observe that there was a certain asymmetry in the funnel plot, indicating that there is some degree of publication bias in the literature. However, the number of studies included was only ten, and the funnel plots may not be very reliable. More over, Egger's test revealed that there was no significant difference in the ORs of AEs in our study $(Z=1.58 ; P=0.46)$.

\section{Discussion}

To the best of our knowledge, this study is the first meta-analysis to evaluate the analgesic efficacy and tolerability of surgery patients receiving oxycodone versus fentanyl for IV-PCA. In the current metaanalysis including data from 600 patients, we found that postoperative intravenous oxycodone combination PCA strategies lead to lower postoperative pain intensity and lower postoperative opioid consumption compared to the fentanyl, although it may increase the incidence of some adverse events. These findings of this meta-analysis suggest that oxycodone IV-PCA provided superior pain relief than fentanyl IV-PCA.

Oxycodone is a potent and highly selective agonist, and acts mainly on post surgical pain control. ${ }^{24}$ It has been confirmed that the onset time of IV oxycodone is 2 to 3 minutes after injection and has a longer duration of action about 4 hours 52 minutes after a short course of treatment. ${ }^{25}$ Previous meta-analysis have concentrated on the use of oxycodone for moderate-serve cancer-related pain or acute preoperative analgesia. ${ }^{26}$ The current meta-analysis was aimed to reveal the postoperative analgesic and tolerant effects of oxycodone and fentanyl for IV-PCA in surgery patients. In this meta-analysis, patient-reported pain intensity was found to be more slightter in oxycodone group at 4, 8, 12 and $24 \mathrm{~h}$ compared to the fentanyl group for IV-PCA. Because of the negative WMD in NRS scores both at rest and movement between oxycodone and fentanyl for IV-PCA, it can therefore be deduced that oxycodone is likely to improve pain compared with fentanyl in patients using IV-PCA. 
A great deal of evidence has proved that oxycodone was more effective for visceral pain treatment than other opioids. ${ }^{27}$ Kalso et al made a comparison of analgesic effect between oxycodone and morphine for patients with laparoscopic cholecystectomy, and it was observed that less oxycodone was needed for the pain relief than morphine. ${ }^{28}$ We speculated that this finding regarding to the oxycodone's mechanism of action. It has been confirmed that oxycodone has high affinity for both central p-receptor and kappa(k)opioid receptors on peripheral nerves, which may be a key factor for modulation of visceral pain. Thus, oxycodone is a scientific and effective drug for postoperative analgesia, especially used for relieving visceral pain. ${ }^{29,30}$

When the anagesic effects of two patient-controlled opioids are compared, it is important to determine the PCA dosage regimens of the two opioids because they affect the efficacy of the analgesia. The results of our meta-analysis also found that there was a significant lower PCA consumption over 48 hours (WMD of $12.11 \mathrm{ml}$ ) in patients administrated with oxycodone compared to the fentanyl, and it needed less postoperative rescue analgesia. Further more, it has been reported that the cost of oxycodone is notablely superior to that of fentanyl in the pharmacoeconomics; the IV-PCA using fentanyl (fentanyl: 16,167 Korean Won, vs oxycodone: 9,824 Korean Won) was much expensive than the IV-PCA using oxycodone (by about $40 \%) .{ }^{17}$

Our meta-analysis of postoperative patient satisfaction in IV-PCA over 48 hours after the surgery showed that there was no statistically significant difference between the group oxycodone and fentanyl group. The above data may suggest that although the oxycodone for IV-PCA showed better effects in postoperative analgesia than the fentanyl alone, the more frequent incidenceof side effects experienced in oxycodone group decreased the satisfaction of patients. ${ }^{31,32}$

Our meta-analysis demonstrated that the oxycodone for IV-PCA use might significantly increase the risk of PONV and dizziness(all $P<0.05$ ) than fentanyl. The possible mechanisms of opioid-induced nausea or vomiting are not incompletely understood, there are two possible explanations for our results: (1) the consumption low-dose opioid drugs by receiving treatment of fentanyl decreased the risk of side effects associated with opioid drugs; (2) the fentanyl's antiemetic characteristics, due to the decrease of norepinephrine activity and sedation effect, may explain why caused lower incidence of PONV. However, there are no reports to date about the details of perioperative antiemetic and sedative prophylaxis.

The opioids widely administered for IV-PCA are full u-receptor-binding agonists and no analgesic ceiling were observed in these agents. However, a clinical ceiling set to prevent AEs such as respiratory depression, which may limit the use of entire required dose for appropriate pain control. Therefore, Ketorolac, a nonsteroidal anti-inflammatory drug (NSAID), has been commonly added to IV-PCA solution to reduce opioid consumption and opioid related side effects. ${ }^{35}$ Furthermore, some studies have shown that local anesthetics and cyclooxygenase (COX)-2 inhibitors can effectively reduce the movementevoked pain by preventing central sensitization. ${ }^{36}$ In addition, combination ketorolac with IV-PCA mixture was reported to shorten the postoperative recovery period in patients with colorectal surgery. ${ }^{37}$ The same 
goes for the included studies of our meta-analysis, ketorolac was mixed to the IV-PCA for the purpose of enhancing the analgesic effects provided by opioids while decreasing their side reactions. ${ }^{17,20-22}$

Finally, a frequent problem is that mixing 2 or more injections together in infusion solutions induced physical changes and chemical degration of ingredients could cause precipitation or crystallization, thus the efficacy was discounted. ${ }^{38}$ There are many clinical studies have evaluated the efficacy of ketorolac or dexmedetomidine as adjuncts to oxycodone IV-PCA to provide superior pain relief, but still little or no information is available about the physical or chemical changes of these analgesic mixtures. ${ }^{39}$ Therefore, it is suggested that the clinicians evaluate the compatibility and stability of the binary admixtures of opioid or non-opioid-oxycodone in future to ensure the safety and efficacy of oxycodone IV-PCA. ${ }^{40}$

Of course this meta-analysis, the course of the paper had some limitations. First, the number of studies included in the meta-analysis is small(only 8 RCTs), and the sample size of patients in our study was also insufficient, potentially making the conclusions less convincing. Second, pain perception is individual and is impacted by parturients' tolerance, progression of labor, human influences(e.g., psychological influence from doctors or nurses), analgesic dose, and other factors. Thus, the pain score by itself may not reflect pain relief efficacy. Third, we observed significant heterogeneities in analytical indicators such as NRS scores and cumulative PCA dose over 48hours; therefore, these findings must be assessed with caution. Furthermore, there may have been publication bias; it could not be completely depended on the funnel plot. Finally, there is a lack of data on the long-term outcomes of the effect of oxycodone and fentanyl for PCIA in the treatment of chronic pain. Therefore, more large-scale and high-quality RCTs are needed to collected to summarize and analyze the data to make the conclusion more reliable.

\section{Conclusion}

In summary, our systemic review and meta-analysis initially demonstrated that PCA-based drug administration, oxycodone IV-PCA showed a better analgesic effect in postoperative analgesia compared with fentanyl in spite of higher rate of PONV. However, all the clinical trials involved were of small samples without blind methods, their results may show some uncertainties. We urgently hope large-scale, high-quality, double-blinded, and multi-centered RCTs will be performed in the future to further confirm its efficacy and safety.

\section{Abbreviations}

PCA-patient-controlled analgesia, RCTs-randomized controlled trials, NRS-numerical rating scale, PONVpostoperative nausea and vomiting, AEs-adverse events, IV-intravenous, MFDS-Ministry of Food and Drug Safety.

\section{Declarations}

\section{Ethics approval and consent to participate}


This study was approved by the Institutional Review Board and performed at the Renmin Hosipital of Wuhan University.

\section{Consent for publication}

Not applicabe.

\section{Availability of data and material}

The datasets supporting the conclusions of this article are included in themanuscript.

\section{Competing interests}

The authors report no conflicts of interest in this work.

\section{Funding}

There was no funding.

\section{Authors' contributions}

Chen P was involved in the protocol design, analysis and or interpretation of data, writing and editing the manuscript. Chen FC and Lei JX contributed to the collection and analysis of data including the statistical analysis. Chen P prepared the first draft. Zhou BH was approved this project. All authors were involved in subsequent editing and agreed on the final version.

\section{Acknowledgements}

None

\section{References}

1. Shafie L, Barghi H, Parirokh M, et al. Postoperative Pain following Pulpotomy of Primary Molars with Two Biomaterials: A Randomized Split Mouth Clinical Trial. Iran Endod J 2017, 12(1):10-14. 2. Rehberg B, Mathivon S, Combescure C, et al. Prediction of Acute Postoperative Pain Following Breast Cancer Surgery Using the Pain Sensitivity Questionnaire: A Cohort Study. Clin J Pain 2017, 33(1):57-66. 3. Binhas M, Egbeolamartial J, Kluger M D, et al. Opioids and nonopioids for postoperative pain control in patients with chronic kidney disease. J Opioid Manag 2017, 13(1):17-25. 4. Seagle B L, Miller E S, Strohl A E, et al. Transversus abdominis plane block with liposomal bupivacaine compared to oral opioids alone for acute postoperative pain after laparoscopic hysterectomy for early endometrial cancer: a cost-effectiveness analysis. Gynecol Oncol 2017, 4(1):12-20. 5. Daily L R, Gordon J, Ulm M, et al. Postoperative opioids: Are physicians prescribing more than what is necessary for adequate pain control?. Gynecol Oncol 2017, 145(1):168-169. 6. Wang X, Wang K, Wang B, et al. Effect of Oxycodone Combined With Dexmedetomidine for Intravenous Patient-Controlled Analgesia After Video-Assisted Thoracoscopic 
Lobectomy. J Cardiothor Vasc An 2016, 30(4):1015-1021. 7. Yeying G, Liyong Y, Yuebo C, et al. Thoracic paravertebral block versus intravenous patient-controlled analgesia for pain treatment in patients with multiplerib fractures. J Int Med Res 2017, 45(6):2085-2091. 8. Kim Y K, Ji H L, Kang S H, et al. The effects of epidural versus intravenous patient-controlled analgesia on postoperative outcomes in elderly patients who have undergone gastrectomy: a retrospective trial. Anesth Pain Med 2017, 12(4):363-370. 9. Van d S R, Roozekrans M, Olofsen E, et al. Influence of Ethanol on Oxycodone-induced Respiratory Depression: A Dose-escalating Study in Young and Elderly Individuals. Anesthesiology 2017, 126(3):534-542. 10. Välitalo P, Kokki M, Ranta V P, et al. Maturation of Oxycodone Pharmacokinetics in Neonates and Infants: a Population Pharmacokinetic Model of Three Clinical Trials. Pharm Res 2017, 34(5):1-9. 11. Emery M A, Shawn Bates M L, Wellman P J, et al. Hydrocodone is More Effective than Morphine or Oxycodone in Suppressing the Development of Burn-Induced Mechanical Allodynia. Pain Med 2017, 18(11):2170-2180. 12. Kim D K, Yoon S H, Kim J Y, et al. Comparison of the Effects of Sufentanil and Fentanyl Intravenous Patient Controlled Analgesia after Lumbar Fusion. J Korean Neurosurg Soc 2017, 60(1):54-59. 13. Na Y K, Kwon T D, Bai S J, et al. Effects of dexmedetomidine in combination with fentanyl-based intravenous patient-controlled analgesia on pain attenuation after open gastrectomy in comparison with conventional thoracic epidural and fentanyl-based intravenous patient-controlled analgesia. Int J Med Sci 2017, 14(10):951-960. 14. Lindley P, Li D, Danesi H, et al. Meta-analysis of the Ease of Care From a Patients' Perspective Comparing Fentanyl Iontophoretic Transdermal System Versus Morphine Intravenous Patient-Controlled Analgesia in Postoperative Pain Management. J Perianesth Nurs 2017, 32(4):320-328. 15. Viscusi E R, Grond S, Ding L, et al. A comparison of opioid-related adverse events with fentanyl iontophoretic transdermal system versus morphine intravenous patient-controlled analgesia in acute postoperative pain. Pain Manag 2016, 6(1):19-24. 16. Jung K W, Kang H W, Park C H, et al. Comparison of the Analgesic Effect of Patient-controlled Oxycodone and Fentanyl for Pain Management in Patients Undergoing Colorectal Surgery. Clin Exp Pharmmacol P 2016, 43(8):745-752. 17. Nan-Seol K, Kang K S, Hyeon Y S, et al. A comparison of oxycodone and fentanyl in intravenous patient-controlled analgesia after laparoscopic hysterectomy. Korean J Anesthesiol 2015, 68(3):261-266. 18. Park J H, Lee C, Shin Y, et al. Comparison of oxycodone and fentanyl for postoperative patient-controlled analgesia after laparoscopic gynecological surgery. Korean J Anesthesiol 2015, 68(2):153-158. 19. Dong K S, Chang J L, Jin S K. A comparison of oxycodone and fentanyl in the management of early postoperative pain and for patient-controlled analgesia after total abdominal hysterectomy. Anesth Pain Med 2016, 11(2):176-181. 20. Ding, Zhen, Wang, et al. Efficacy and tolerability of oxycodone versus fentanyl for intravenous patientcontrolled analgesia after gastrointestinal laparotomy. Medicine 2016, 95(39):4943-4947. 21. Kim N S, Lee J S, Park S Y, et al. Oxycodone versus fentanyl for intravenous patient-controlled analgesia after laparoscopic supracervical hysterectomy: A prospective, randomized, double-blind study. Medicine 2017, 96(10):6286-6291. 22. Hwang B Y, Kwon J Y, Kim E. Oxycodone vs. fentanyl patient-controlled analgesia after laparoscopic cholecystectomy. Int J Med Sci 2014, 11(7):658-62. 23. Yang S A, Kim K S, Kang H Y. Oxycodone vs. fentanyl in the treatment of early post-operative pain after total knee replacement: randomized controlled trial. Anesth Pain Med 2016, 11(4):349-353. 24. Webster L, Henningfield J, Buchhalter A R, et al. Human Abuse Potential of the New Opioid Analgesic Molecule NKTR-181 Compared with Oxycodone. Pain Med 2018, 19(2):307-318. 25. Wang Y M, Liu Z W, Liu J L, et al. Efficacy and 
tolerability of oxycodone in moderate-severe cancer-related pain: A meta-analysis of randomized controlled trials. Exp Ther Med 2012, 4(2):249-254. 26. King S J, Reid C, Forbes K, et al. A systematic review of oxycodone in the management of cancer pain. Palliat Med 2011, 25(5):454-470. 27. Jr P J, Seowchoen F, Wexner S D, et al. Perspectives on Intravenous Oxycodone for Control of Postoperative Pain. Pain Practice, 2016, 16(7):924-934. 28. Kalso E, Pöyhiä R, Onnela P, et al. Intravenous morphine and oxycodone for pain after abdominal surgery. Acta Anaesth Scand 2010, 35(7):642-646. 29. Backonja M, Webster L R, Setnik B, et al. Intravenous abuse potential study of oxycodone alone or in combination with naltrexone in nondependent recreational opioid users. Am J Drug Alcohol Ab 2016, 42(5):539-549. 30. Creamer F, Balfour A, Nimmo S, et al. Randomized open-label phase II study comparing oxycodonenaloxone with oxycodone in early return of gastrointestinal function after laparoscopic colorectal surgery. Br J Surg 2016, 104(1):42-51. 31. Somerville N J, O'Donnell J, Gladden R M, et al. Characteristics of Fentanyl Overdose-Massachusetts, 2014-2016. Mmwr Morb Mortal Wkly Rep 2017, 66(14):382-386. 32. Nicholson B D. Economic and clinical burden of opioid-induced nausea and vomiting. J Postgrad Med 2017, 129(1):111-117. 33. Raffa R B, Colucci R, Pergolizzi J V. The effects of food on opioid-induced nausea and vomiting and pharmacological parameters: a systematic review. J Postgrad Med 2017, 129(7):698-708. 34. Theresa Mallick-Searle MS RN-BC ANP-BC, Mechele Fillman MSN NP-BC. The pathophysiology, incidence, impact, and treatment of opioid-induced nausea and vomiting. J Am Assoc Nurse Pra 2017, 29(11):704-710. 35. Murdoch J, Ramsey G, Day A G, et al. Intraperitoneal ketorolac for post-cholecystectomy pain: a double-blind randomized-controlled trial. Can J Anaesth 2016, 63(6):701708. 36. Praveen R, Thakur S, Kirthiga M. Comparative Evaluation of Premedication with Ketorolac and Prednisolone on Postendodontic Pain: A Double-blind Randomized Controlled Trial. J Endod 2017, 43(5):667-673. 37. Lim K I, Liu C K, Chen C L, et al. Transitional Study of Patient-Controlled Analgesia Morphine With Ketorolac to Patient-Controlled Analgesia Morphine With Parecoxib Among Donors in Adult Living Donor Liver Transplantation: A Single-Center Experience. Transplantation Proc 2016, 48(4):1074-1076. 38. Chen F C, Zhu J, Li B, et al. Stability of tramadol with three 5-HT3receptor antagonists in polyolefin bags for patient-controlled delivery systems. Drug Des Dev Ther 2016, 10(1):1869-1875. 39. Suman P, Rao T S, Suman P, et al. High Performance Liquid Chromatographic Method for Simultaneous Quantification of Naloxone and Oxycodone. Asian J Chem 2017, 29(11):24972500. 40. Houlihan S, Decarie D, Benes C, et al. Magnocaine: Physical Compatibility and Chemical Stability of Magnesium Sulphate and Lidocaine Hydrochloride in Prefilled Syringes. J Obstet Gynaecol Can 2016, 38(10):936-944.e3.

\section{Figure And Table Legends}

Figure 1. Flow diagram of the study selection process.

Figure 2. Risk of bias percentile chart.

Figure 3. Risk of bias assessment of randomized controlled trials included in meta-analysis. 
Figure 4. Forest plots analysis of the NRS scores at rest for oxycodone versus fentanyl.

Figure 5. Forest plots analysis of the NRS scores at movement for oxycodone versus fentanyl.

Figure 6. Forest plots analysis of cumulative PCA dose over 48hours for oxycodone versus fentanyl.

Figure 7. Forest plots analysis of incidence of PONV for oxycodone versus fentanyl.

Figure 8. Forest plots analysis of patient satisfaction for oxycodone versus fentanyl.

Figure 9. Forest plots analysis of main adverse events for oxycodone versus fentanyl.

Figure 10. The funnel plot of publication bias

Table. 1 Baseline characteristics of trials included in the meta-analysis

\section{Figures}

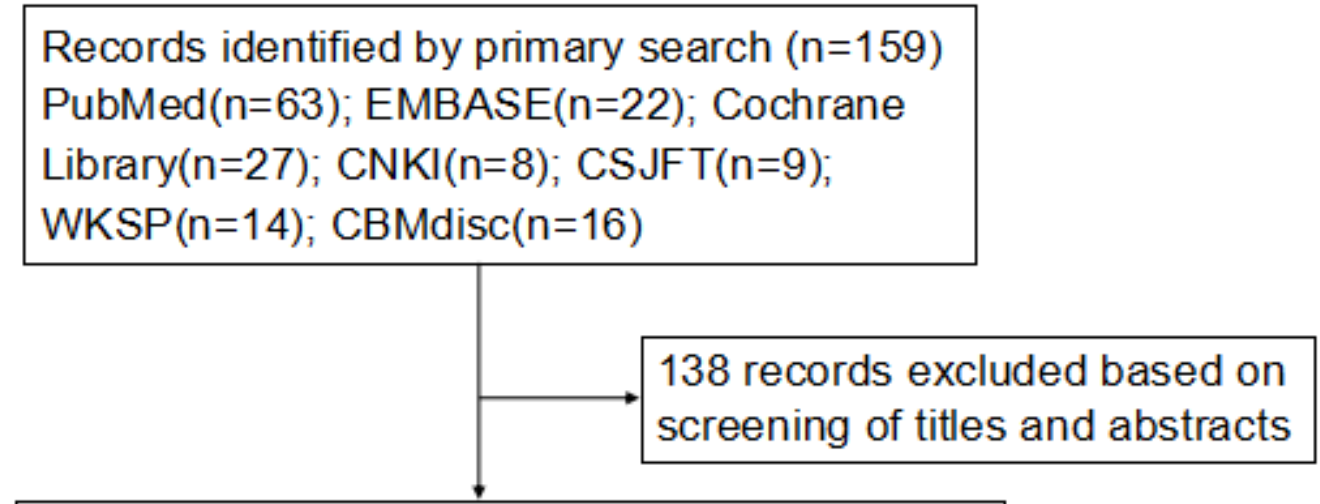

21 potentially relevant full text articles included for analysis

13 trails excluded data missing $(n=5)$ duplicated reports $(n=4)$ ineligible patients $(n=4)$

Studies included in meta-analysis $(n=8)$

\section{Figure 1}

Flow diagram of the study selection process. 


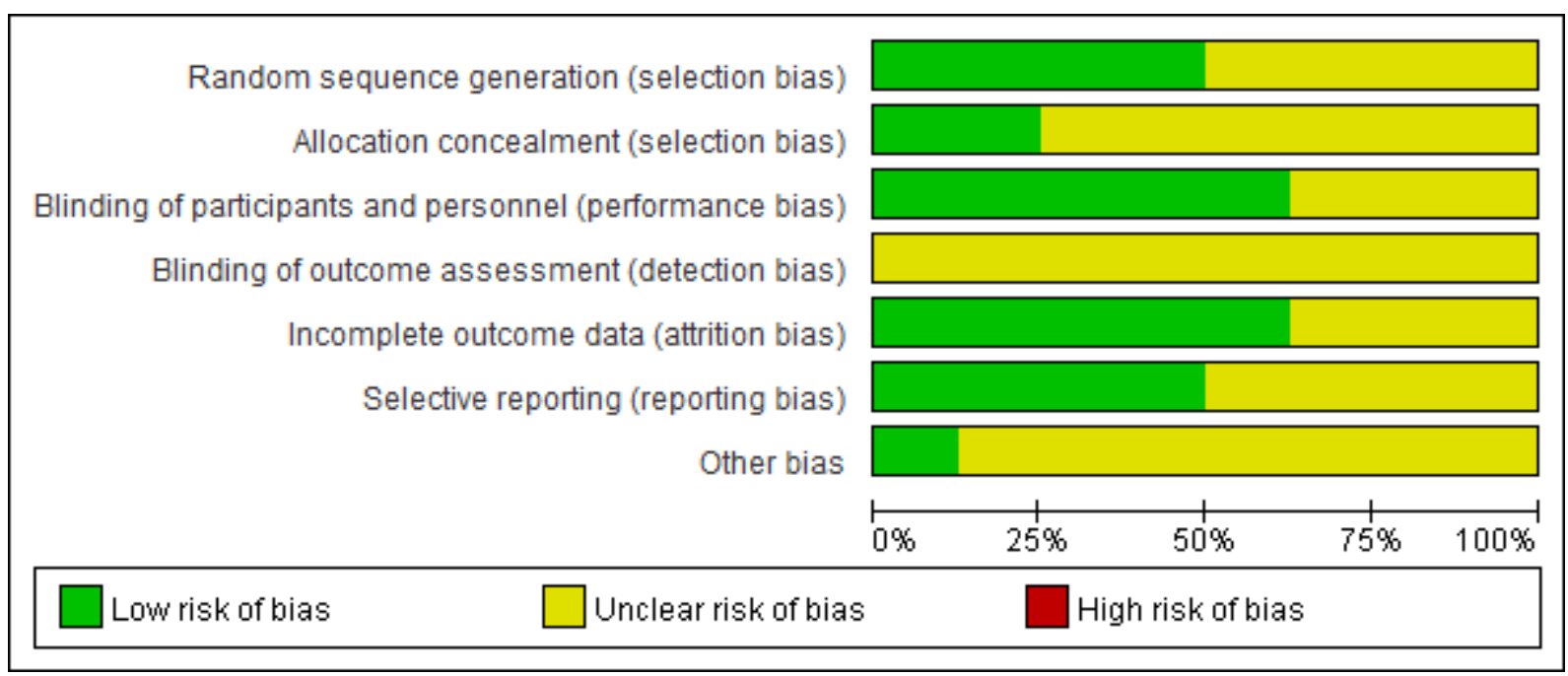

Figure 2

Risk of bias percentile chart.

\begin{tabular}{|c|c|c|c|c|c|c|c|}
\hline & 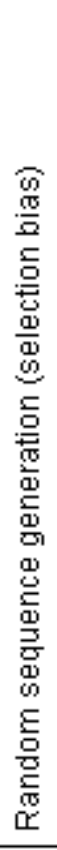 & 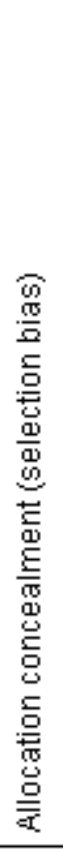 & 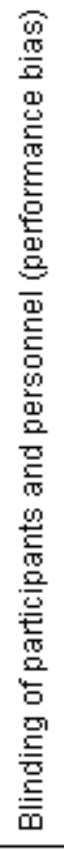 & 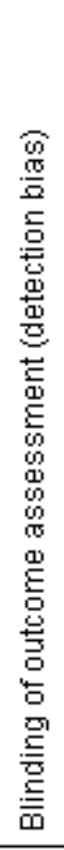 & 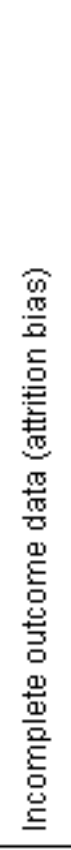 & 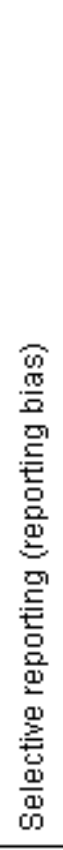 & 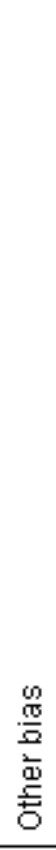 \\
\hline Dong K S(2016) & $?$ & + & + & $?$ & $?$ & $?$ & $?$ \\
\hline Hwang $B Y(2017)$ & + & + & + & $?$ & $?$ & $?$ & $?$ \\
\hline Jung KW(2016) & $?$ & $?$ & $?$ & $?$ & $\oplus$ & + & + \\
\hline Kim N S(2017) & $?$ & $?$ & + & $?$ & $\oplus$ & + & $?$ \\
\hline Nan-Seol K(2015) & + & $?$ & $?$ & $?$ & $?$ & $?$ & $?$ \\
\hline Park.J H(2015) & + & $?$ & $\oplus$ & $?$ & $\odot$ & + & $?$ \\
\hline Yang $S A(2016)$ & ? & ? & $?$ & ? & $\odot$ & + & $?$ \\
\hline Zhen D(2016) & + & $?$ & + & $?$ & + & $?$ & $?$ \\
\hline
\end{tabular}




\section{Figure 3}

Risk of bias assessment of randomized controlled trials included in meta-analysis.

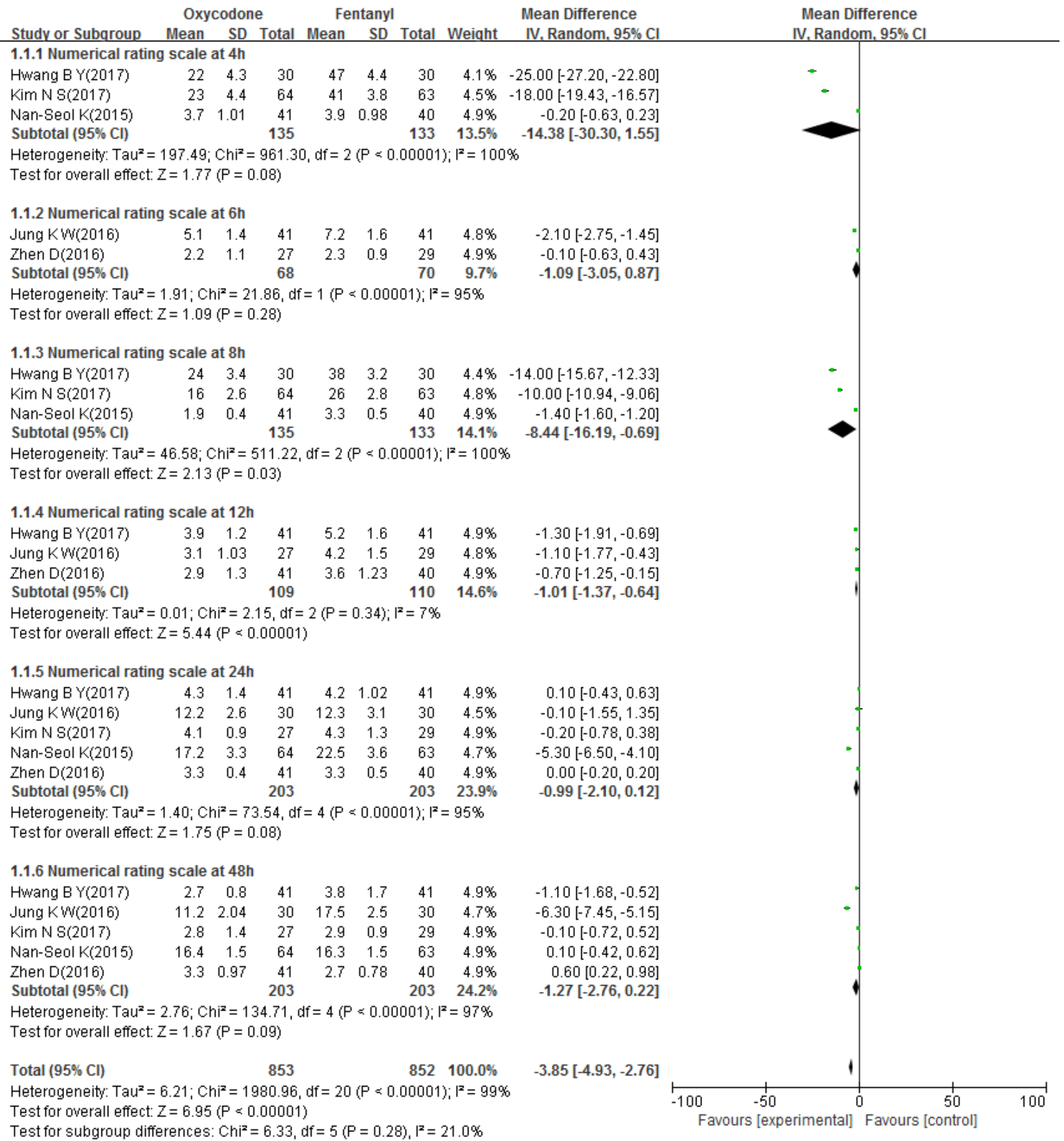

\section{Figure 4}

Forest plots analysis of the NRS scores at rest for oxycodone versus fentanyl. 


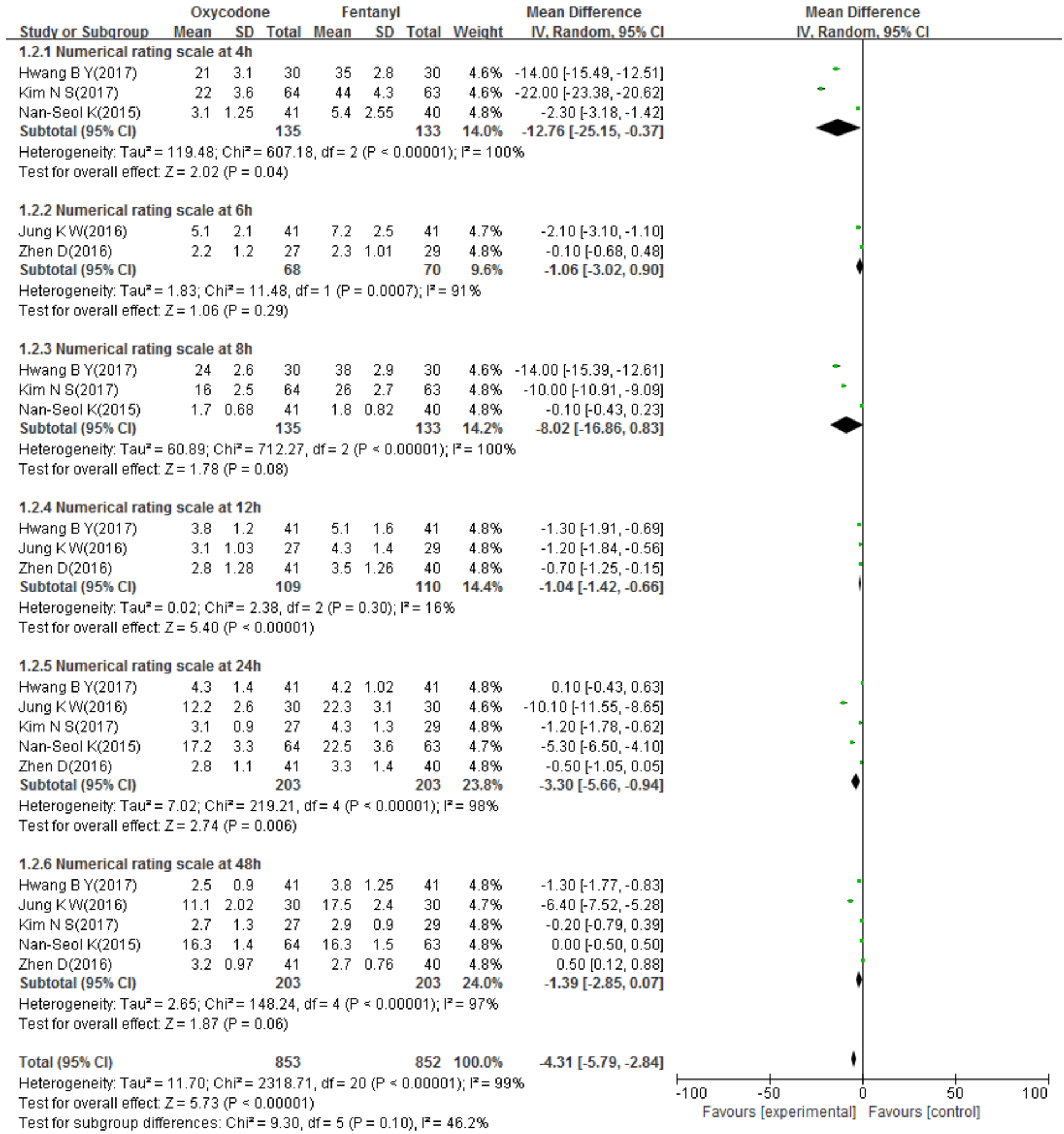

\section{Figure 5}

Forest plots analysis of the NRS scores at movement for oxycodone versus fentanyl. 


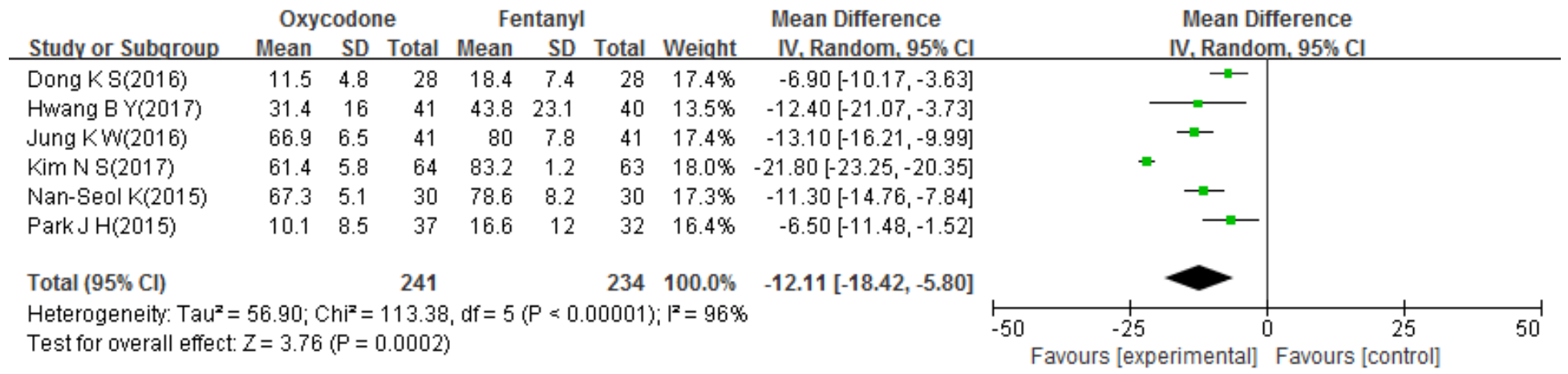

Figure 6

Forest plots analysis of cumulative PCA dose over 48hours for oxycodone versus fentanyl.

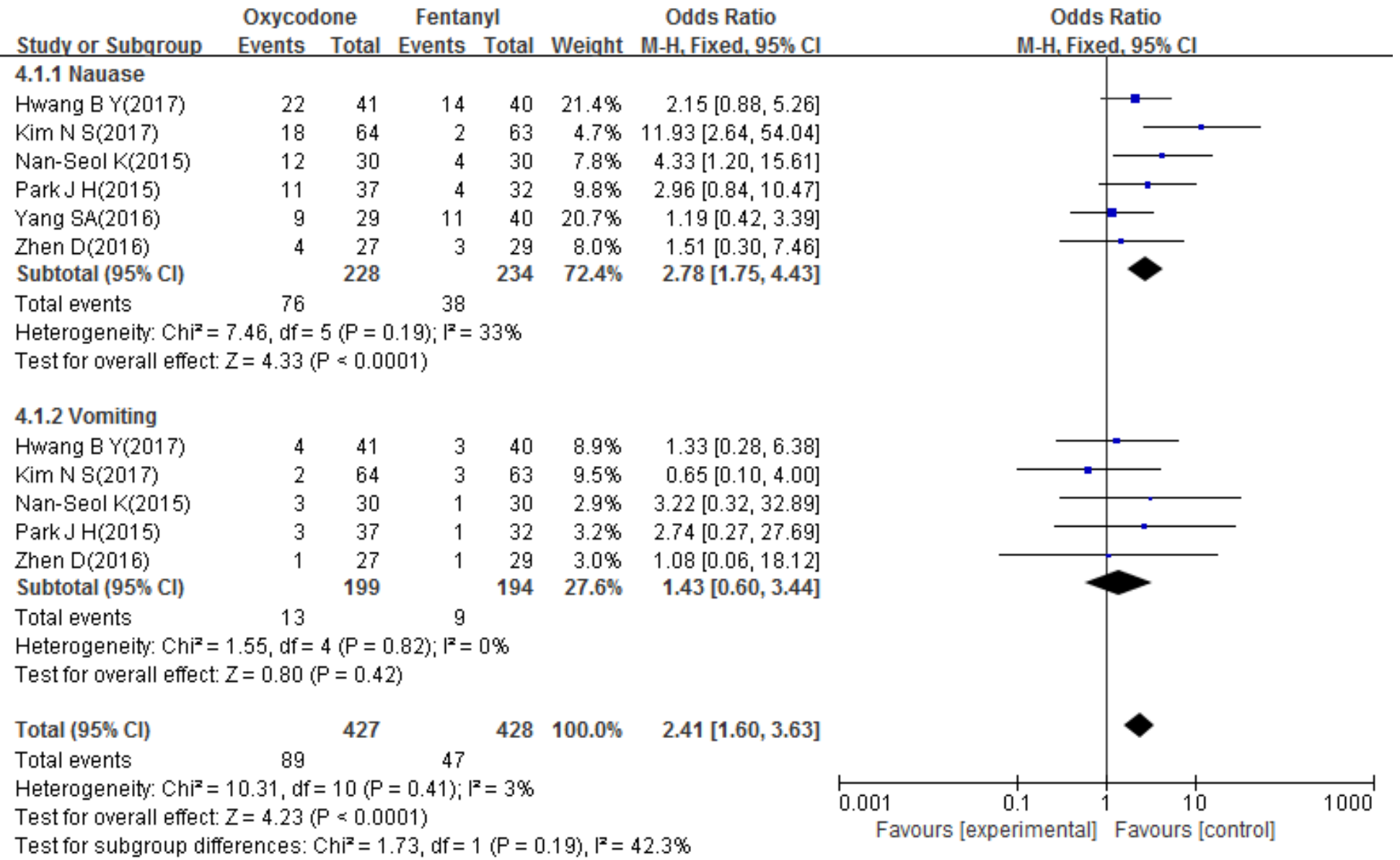

\section{Figure 7}

Forest plots analysis of incidence of PONV for oxycodone versus fentanyl. 
Oxycodone Fentanyl Odds Ratio

Study or Subqroup Events Total Events Total Weight M-H, Random, 95\% Cl M-H, Random, $95 \% \mathrm{Cl}$

Hwang B Y(2017)

Kim N S(2017)

Nan-Seol K(2015)

Zhen $\mathrm{D}(2016)$

Total $(95 \% \mathrm{Cl})$

Total events

$\begin{array}{rrrrr}41 & 41 & 37 & 40 & 19.9 \% \\ 54 & 64 & 61 & 63 & 30.7 \% \\ 26 & 30 & 30 & 30 & 20.1 \% \\ 25 & 27 & 24 & 29 & 29.3 \% \\ & 162 & & 162 & 100.0 \% \\ 146 & & 152 & & \end{array}$

Heterogeneity: $\mathrm{Tau}^{2}=2.53 ; \mathrm{Chi}^{2}=9.35, \mathrm{df}=3(\mathrm{P}=0.03) ; \mathrm{I}^{2}=68 \%$

Test for overall effect: $Z=0.32(P=0.75)$
$7.75[0.39,154.96]$

$0.18[0.04,0.84]$

$0.10[0.00,1.88]$

$2.60[0.46,14.73]$

$0.73[0.11,5.04]$

\section{Figure 8}

Forest plots analysis of patient satisfaction for oxycodone versus fentanyl.

Favours [experimental] Favours [control]

\begin{tabular}{|c|c|c|c|c|c|c|}
\hline \multirow[b]{2}{*}{ Study or Subqroup } & \multicolumn{2}{|c|}{ Oxycodone } & \multicolumn{2}{|c|}{ Fentanyl } & \multirow{2}{*}{ Weiqht } & \multirow{2}{*}{$\begin{array}{c}\text { Odds Ratio } \\
\text { M-H, Fixed, } 95 \% \mathrm{Cl}\end{array}$} \\
\hline & Events & Total & Events & Total & & \\
\hline \multicolumn{7}{|l|}{ 4.2.1 Dizziness } \\
\hline Kim N S(2017) & 8 & 30 & 6 & 30 & $8.9 \%$ & $1.45[0.44,4.86]$ \\
\hline Nan-Seol K(2015) & 13 & 37 & 3 & 32 & $4.2 \%$ & $5.24[1.33,20.54]$ \\
\hline Park J H(2015) & 6 & 27 & 4 & 29 & $6.1 \%$ & $1.79[0.44,7.18]$ \\
\hline Zhen D(2016) & 43 & 64 & 16 & 63 & $10.7 \%$ & $6.01[2.78,13.00]$ \\
\hline Subtotal $(95 \% \mathrm{Cl})$ & & 158 & & 154 & $30.0 \%$ & $3.69[2.17,6.26]$ \\
\hline Total events & 70 & & 29 & & & \\
\hline
\end{tabular}

Heterogeneity: $\mathrm{Chi}^{2}=5.13, \mathrm{df}=3(\mathrm{P}=0.16) ; \mathrm{I}^{2}=41 \%$

Test for overall effect: $Z=4.83$ ( $<<0.00001$ )

\subsubsection{Headache} Hwang B Y(2017) Kim N S(2017) Nan-Seol K(2015)

Park.J H(2015)

Subtotal $(95 \% \mathrm{Cl})$

Total events

Heterogeneity: $\mathrm{Chi}^{2}=3.39, \mathrm{df}=3(\mathrm{P}=0.34) ; \mathrm{I}^{2}=11 \%$

Test for overall effect: $Z=0.93(P=0.35)$

\subsubsection{Pruritis}

Hwang $B Y(2017)$

Kim N S(2017)

Nan-Seol K(2015)

Subtotal $(95 \% \mathrm{Cl})$

Total events

\begin{tabular}{rrrr}
4 & 30 & 4 & 30 \\
7 & 37 & 1 & 32 \\
8 & 64 & 9 & 63 \\
2 & 41 & 1 & 40 \\
& 172 & & 165 \\
21 & \multicolumn{5}{c}{15} \\
df $=3(P=0.34) ;\left.\right|^{2}=11 \%$ \\
$93(P=0.35)$
\end{tabular}

Heterogeneity: $\mathrm{Chi}^{2}=3.06, \mathrm{df}=2(\mathrm{P}=0.22) ; \mathrm{I}^{2}=35 \%$

Test for overall effect: $Z=1.05(P=0.29)$

$\begin{array}{lrrr}4 & 30 & 3 & 30 \\ 9 & 64 & 3 & 63 \\ 1 & 41 & 3 & 40 \\ & 135 & & 133 \\ 14 & 9 & \\ d f=2 & (P=0.22) ;\left.\right|^{2}= & 35 \% \\ .05 & (P=0.29)\end{array}$

$1.00[0.23,4.43]$

$7.23[0.84,62.38]$

$0.86[0.31,2.38]$

$2.00[0.17,22.97]$

$1.40[0.69,2.83]$
Odds Ratio M-H, Fixed, $95 \% \mathrm{Cl}$

\subsection{4 sedation}

ParkJ H(2015)

Yang SA(2016)

Zhen D(2016)

Subtotal $(95 \% \mathrm{Cl})$

Total events

$\begin{array}{rr}5.3 \% & 1.38[0.28,6.80] \\ 5.3 \% & 3.27[0.84,12.71] \\ 6.0 \% & 0.31[0.03,3.10] \\ 16.6 \% & 1.60[0.67,3.81]\end{array}$

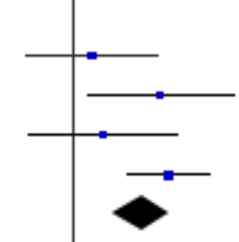

$1.34[0.49,3.64]$

$7.36[0.34,159.38]$

$1.34[0.47,3.83]$

$1.52[0.76,3.04]$

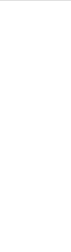

Heterogeneity: $\mathrm{Chi}^{2}=1.13, \mathrm{df}=2(\mathrm{P}=0.57) ; \mathrm{I}^{2}=0 \%$

Test for overall effect: $Z=1.18(P=0.24)$

Total $(95 \% \mathrm{Cl})$

Total events

Heterogeneity: $\mathrm{Ch}^{2}=19.92, \mathrm{df}=13(\mathrm{P}=0.10) ; \mathrm{I}^{2}=35 \%$

Test for overall effect: $Z=4.55$ ( $P<0.00001$ )

Test for subgroup differences: $\mathrm{Ch}^{2}=6.85, \mathrm{df}=3(\mathrm{P}=0.08), \mathrm{I}^{2}=56.2 \%$ 
Figure 9

Forest plots analysis of main adverse events for oxycodone versus fentanyl.

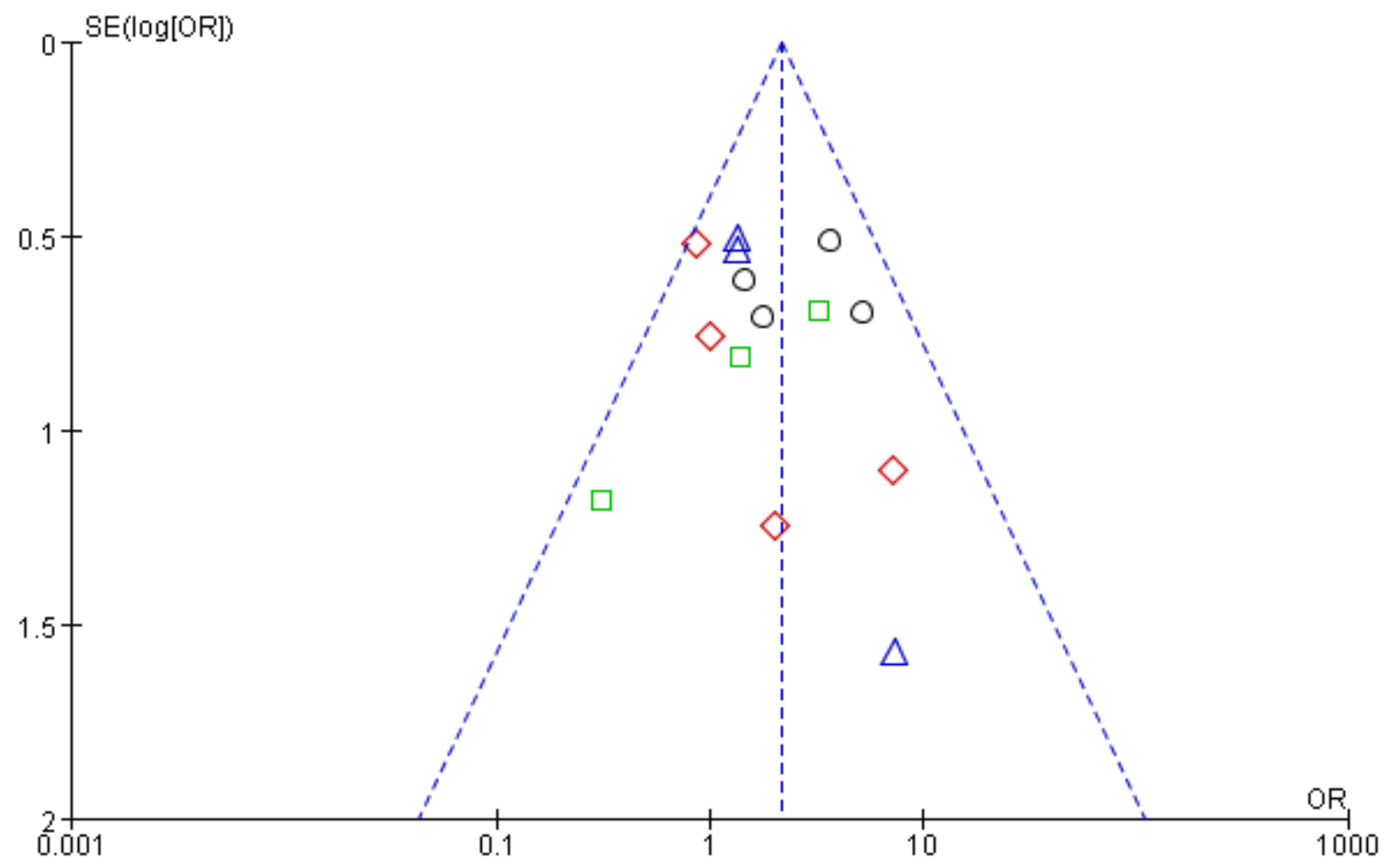

${ }^{\text {Subgroups }} \diamond_{\text {Headache }} \square$ Pruritis $\Delta$ sedation

\section{Figure 10}

The funnel plot of publication bias

\section{Supplementary Files}

This is a list of supplementary files associated with this preprint. Click to download.

- Table1.pdf

- PRISMA2009checklist.pdf 